\title{
TWO NONEQUIVALENT CONDITIONS FOR WEIGHT FUNCTIONS
}

\section{CHARLES FEFFERMAN ${ }^{1}$ AND BENJAMIN MUCKENHOUPT ${ }^{2}$}

ABSTRACT. A nonnegative function on the real line satisfies the condition $A_{\infty}$ if, given $\epsilon>0$, there exists a $\delta>0$ such that if $I$ is an interval, $E \subset I$, and $|E|<\delta|I|$, then $\int_{E} W \leq \epsilon \int_{I} W$. A nonnegative function on the real line satisfies the condition $A$ if for every interval $I, \int_{2 l} W \leq C \int_{I} W$, where $2 I$ is the interval with the same center as $I$ and twice as long, and $C$ is independent of $I$. An example is given of a function that satisfies A but not $\mathrm{A}_{\infty}$.

1. Introduction. The condition $A_{\infty}$ is important in [1], [3] and [5] since for various pairs of operators, $T$ and $U$, there is a constant $C$ such that $\int|T f|^{p} W \leq C \int|U f|^{p} W$ provided $W$ satisfies $A_{\infty}$. In [6] an inequality of this type is proved for $W$ satisfying $A$. The proof in [6] would be much simpler if $W$ were assumed to satisfy $A_{\infty}$. For this reason and the fact that both $A_{\infty}$ and $A$ appear quite naturally in weighted norm inequality problems, it seems to be of interest to give an example of a function that satisfies $A$ but not $A_{\infty}$.

An example of such a function in a dyadic setting was discovered by $D$. Burkholder and communicated to us orally. The dyadic example provided the inspiration for parts of this paper.

If $W$ satisfies the condition $A_{\infty}$, it follows from the results of [4] or the alternate definition of $A_{\infty}$ used in [6] that $W$ satisfies $A$. It is also easy to show this directly as follows. Suppose that $W$ satisfies $A_{\infty}$, and let $\delta$ correspond to $\epsilon=1 / 2$ in the definition of $A_{\infty}$. Let $n$ be the least integer greater than $1 / \delta$, and, given an interval $I$, let $I_{k}$ be the interval with the same center as $I$ and with $\left|I_{k}\right|=(n+k)|I| / n$. By the definition of $\delta$

$$
\int_{I_{k+1}-I_{k}} W \leq \frac{1}{2} \int_{I_{k+1}} W
$$

Received by the editors June 22, 1973.

AMS (MOS) subject classifications (1970). Primary 26A33, 44A15.

1 Supported in part by NSF grant GP 28271.

2 Supported in part by NSF grants GP 20147 and GP 38540. 
Therefore,

$$
\int_{I_{k+1}} W \leq 2 \int_{I_{k}} W
$$

and

$$
\int_{2 I} W=\int_{I_{n}} W \leq 2^{n} \int_{I_{0}} W=2^{n} \int_{I} W
$$

The example of this paper then shows that $A_{\infty}$ is a condition that is strictly stronger than $A$. The example, $W(x)$, developed here is a one dimensional one, but an $n$ dimensional example can be obtained by defining $W_{n}(x)=W\left(x_{1}\right)$, where $x_{1}$ is the first coordinate of $x$. The fact that $W_{n}$ satisfies $A$ but not $A_{\infty}$ in $R^{n}$ follows immediately from the fact that $W$ has this property in $R$.

Throughout this paper the letters $i, j, k, m$ and $n$ will denote integers whether this is stated in the context or not; the letters $a, b, b$ and $x$ will be arbitrary real numbers, and $C$ will denote a positive constant not necessarily the same at each occurrence.

2. Definitions and simple observations. Define

$$
f_{n}(x)=\left\{\begin{array}{rr}
1 / 2, & 0<x \leq 4^{-n-1}, \\
3 / 2, & 4^{-n-1}<x \leq 3 \cdot 4^{-n-1}, \\
1 / 2, & 3 \cdot 4^{n-1}<x \leq 4^{-n},
\end{array}\right.
$$

and to have period $4^{-n}$. An equivalent definition is

$$
f_{n}(x)=1-\left(r_{2 n}(x) r_{2 n+1}(x)\right) / 2
$$

where $r_{n}(x)=\operatorname{sgn}\left[\sin \left(2^{n+1} \pi x\right)\right]$ is the $n$th Rademacher function. For positive $x$ define $n(x)$ to be the integer such that

$$
4^{-n(x)-1}<x \leq 4^{-n(x)}
$$

and define

$$
W(x)=\prod_{k=0}^{2 n(x)} f_{k}(x), \quad 0<x \leq 1
$$

It will be shown that $W(x)$ satisfies condition $A$ but not $A_{\infty}$ on $[0,1]$. If $W(x)$ is defined to be 1 for $x>1$ and if $W(x)$ is defined to be $W(-x)$ for 
negative $x$, it follows trivially from the result on $[0,1]$ that $W(x)$ satisfies the condition $A$ but not $A_{\infty}$ on $(-\infty, \infty)$.

Next define

$$
W_{b}(x)=\prod_{k=0}^{m(x, b)} f_{k}(x),
$$

where $m(x, h)$ is the smaller of $2 n(x)$ and $n(|h|)$. The following elementary observations will be needed; proofs are given after the statements.

1. $\int_{a}^{a+4^{-j}} \Pi_{k=m}^{n} f_{k}(x) d x=4^{-j}$ provided $j \leq m \leq n$.

2. $\Pi_{k=0}^{n} f_{k}(x)$ is constant on the interval $\left(m 4^{-n-1},(m+1) 4^{-n-1}\right]$ for $m$ an integer and on adjacent intervals of this type differs by a factor of 3 , $1 / 3$ or 1 .

3. On $\left(1 / 24^{-n}, 4^{-n}\right], W(x)=3^{k} 2^{-2 n-1}$ on a subset of measure $\left(\begin{array}{c}n+1 \\ k\end{array}\right) 2^{-3 n-2}$.

4. If $0<x<1,-x / 2 \leq h \leq x$ and $0 \leq \theta \leq 1$, then

$$
W_{b}(x) / 324 \leq W_{b}(x+\theta h) \leq 729 W_{b}(x) / 4
$$

Because of periodicity it is sufficient to prove observation 1 for the case $a=0$. On an interval $I_{i}=\left(i 4^{-n},(i+1) 4^{-n}\right], \Pi_{k=m}^{n-1} f_{k}(x)$ is constant, and the integral of $f_{n}(x)$ is $4^{-n}$. Therefore,

$$
\int_{I_{i}} \prod_{k=m}^{n} f_{k}(x) d x=\int_{I_{i}} \prod_{k=m}^{n-1} f_{k}(x) d x .
$$

Adding these for $0 \leq i<4^{n-j}$ shows that

$$
\int_{0}^{4-j} \prod_{k=m}^{n} f_{k}(x) d x=\int_{0}^{4-j} \prod_{k=m}^{n-1} f_{k}(x) d x .
$$

This process then can be repeated to obtain the result.

Observation 2 follows from the fact that $f_{k}(x)$ changes by a factor of 3 at the points $(2 j+1) 4^{-k-1}=(2 j+1) 4^{n-k} 4^{-n-1}$ and these points cannot coincide for different $k$ 's.

For observation 3 , first note that $f_{k}(x)=1 / 2$ for $x$ in $\left(1 / 24^{-n}, 4^{-n}\right]$ and $0 \leq k \leq n-1$. The functions $f_{n}, \cdots, f_{2 n}$ are independently distributed on $\left(1 / 24^{-n}, 4^{-n}\right]$ since each has a full period where the preceding one is constant; this independence also follows immediately from (2.2). Furthermore, 
each of these functions equals $1 / 2$ on half the interval and $3 / 2$ on the other half. Therefore, on $\left(1 / 24^{-n}, 4^{-n}\right], \mathrm{II}_{k=n}^{2 n} f_{k}(x)$ equals $3^{k} 2^{-n-1}$ on a subset of measure $\left(\begin{array}{c}n+1 \\ k\end{array}\right) 2^{-3 n-2}$. Observation 3 follows immediately from this.

To prove observation 4 , fix $x$ and $h$ satisfying the conditions and let $g(y)=\Pi_{k=0}^{m(x, b)} f_{k}(y)$. By observation 2, $g(y)$ changes by at most a factor of 3 on an interval of length $4^{-n(|b|)-1}$. Since $|\theta h| \leq|h| \leq 4^{-n(|b|)}$, $3^{-4} g(x) \leq g(x+\theta h) \leq 3^{4} g(x)$. Since $-x / 2 \leq h \leq x$, the product for $W_{h}(x+\theta h)$ can have at most two more or two fewer $f_{k}$ 's in it than $g(x+\theta h)$ so

$$
2^{-2} g(x+\theta h) \leq W_{b}(x+\theta h) \leq 3^{2} 2^{-2} g(x+\theta h) .
$$

Combining these inequalities and using the fact that $g(x)=W_{b}(x)$ proves observation 4 .

3. W satisfies condition $A$ on $[0,1]$. It will be shown that there are positive constants, $C_{1}$ and $C_{2}$, such that if $0 \leq h \leq b$ and $b+h \leq 1$, then

$$
C_{1} h W_{b}(b) \leq \int_{b}^{b+b} W(x) d x \leq C_{2} h W_{b}(b),
$$

and

$$
C_{1} h W_{b}(b) \leq \int_{b-b}^{b} W(x) d x \leq C_{2} h W_{b}(b)
$$

These show that the integrals of $W$ over two adjacent subintervals of $[0,1]$ of the same size are comparable. This obviously implies condition $A$ by considering the two halves of the interval that is to be doubled.

To prove the second inequality in (3.1) write the integral as

$$
\int_{b}^{b+b} W_{b}(x) \frac{W(x)}{W_{b}(x)} d x
$$

By observation 4, (3.3) is bounded by

$$
C W_{b}(b) \int_{b}^{b+b} \frac{W(x)}{W_{b}(x)} d x ;
$$

as noted in $\$ 1, C$ will denote a positive constant not necessarily the same at each occurrence. By (2.3), (2.4) and (2.5), (3.4) is bounded by

$$
C W_{b}(b) \int_{b}^{b+4-n(b)} \prod_{k=m(b, b)+1}^{2 n(b)} f_{k}(x) d x .
$$


Then observation 1 and the fact that $4^{-n(b)} \leq 4 h$ complete the proof of the right inequality in (3.1). For the left inequality in (3.1), proceed in the same manner, and use the first inequality in observation 4 to show that (3.3) is bounded below by (3.4) with a different positive $C$. Next, (3.4) is bounded below by

$$
C W_{b}(b) \int_{b}^{b+4-n(b)-1} \prod_{k=m(b, b)+1}^{2 n(b)} f_{k}(x) d x
$$

Observation 1 and the fact that $4^{-n(b)-1} \geq h / 4$ complete this part.

If $0<h \leq b / 2$, the inequalities (3.2) follow immediately from (3.1) and property 4 . If $b / 2<h \leq b$, then by the case just observed

$$
\int_{b-b}^{b} W(x) d x \geq \int_{b / 2}^{b} W(x) d x \geq C_{1} \frac{b}{2} W_{b / 2}(b) .
$$

By the definition of $W_{b}$ this is bounded below by $C h W_{b}(b)$, and the first inequality in (3.2) is proved in this case also. Finally, to prove the second inequality in (3.2) if $b / 2<h \leq b$, use the fact that

$$
\int_{b-b}^{b} W(x) d x \leq \int_{0}^{4-n(b)} W(x) d x=\sum_{j=n(b)}^{\infty} \int_{4-j-1}^{4-j} W(x) d x .
$$

Now on $\left[4^{-j-1}, 4^{-j}\right], f_{k}(x)=1 / 2$ for $0 \leq k \leq j-1$ and $f_{j}(x) \leq 3 / 2$. Therefore, by observation 1 ,

$$
\int_{4-j-1}^{4-j} W(x) d x \leq 3 \cdot 2^{-j} \int_{4-j-1}^{4-j} \prod_{k=j+1}^{2 j} f_{k}(x) d x=9 \cdot 2^{-3 j-2}
$$

and by $(3.7)$

$$
\int_{b-b}^{b} W(x) d x \leq C \cdot 2^{-3 n(b)}
$$

Since $b / 2<h \leq b, 4^{-n(b)-2}<h \leq 4^{-n(b)}$, and $n(h)$ equals $n(b)$ or $n(b)+1$. Therefore, $2^{-2 n(b)} \leq 4 \cdot 2^{-2 n(b)}$, and the definitions of $W_{b}$ and $f_{k}$ show that $2^{-n(b)} \leq 2 \Pi_{k=0}^{n(b)} f_{k}(b) \leq 4 W_{b}(b)$. Writing the right side of (3.8) as $C \cdot 2^{-n(b)_{2}-2 n(b)}$ and using these two facts complete the proof of the right inequality in (3.2)

4. $W$ does not satisfy $A_{\infty}$ on $[0,1]$. This will be shown by producing a sequence of intervals, $I_{n}$, and a sequence of sets, $E_{n}$, such that 


$$
E_{n} \subset I_{n}, \quad \lim _{n \rightarrow \infty} \frac{\left|E_{n}\right|}{\left|I_{n}\right|}=0 \text { and } \lim _{n \rightarrow \infty} \int_{E_{N}} W / \int_{I_{n}} W=1
$$

To do this let $I_{n}=\left[1 / 24^{-n}, 4^{-n}\right]$ and let $E_{n}$ be the subset of $I_{n}$ where $W(x)>3^{2 n / 3} 2^{-2 n-1}$. By observation 3 ,

$$
\frac{\left|E_{n}\right|}{\left|I_{n}\right|}=\sum_{k=[2 n / 3]+1}^{n+1}\left(\begin{array}{c}
n+1 \\
k
\end{array}\right)\left(\frac{1}{2}\right)^{k}\left(\frac{1}{2}\right)^{n+1-k}
$$

and

$$
\int_{E_{n}} W / \int_{I_{n}} W=\sum_{k=\{2 n / 3]+1}^{n+1}\left(\begin{array}{c}
n+1 \\
k
\end{array}\right)\left(\frac{3}{4}\right)^{k}\left(\frac{1}{4}\right)^{n+1-k},
$$

where $[2 n / 3]$ denotes the greatest integer less than or equal to $2 n / 3$. That the limits of these two ratios are 0 and 1 respectively follows immediately from the fact $[2,(16.4 .6)$, p. 200] that if $0<p<1, q=1-p$ and $a<b$, then

$$
\lim _{n \rightarrow \infty} \sum_{n p+a(n p q)^{1 / 2}<k \leq n p+b(n p q)^{1 / 2}}\left(\begin{array}{l}
n \\
k
\end{array}\right) p^{k} q^{n-k}=(2 \pi)^{-1 / 2} \int_{a}^{b} e^{-x^{2} / 2} d x .
$$

\section{REFERENCES}

1. R. Coifman and C. Fefferman, Weighted norm inequalities for maximal functions and singular integrals, Studia Math. (to appear).

2. H. Cramér, Mathematical methods of statistics, Princeton Math. Series, vol. 9, Princeton Univ. Press, Princeton, N. J., 1946. MR 8, 39.

3. R. Gundy and R. Wheeden, Weighted integral inequalities for the nontangential maximal function, Lusin area integral and Walsh-Paley series, Studia Math. (to app ear).

4. B. Muckenhoupt, The equivalence of two conditions for weight functions, Studia Math. (to appear).

5. B. Muckenhoupt and R. Wheeden, Weighted norm inequalities for fractional integrals, Trans. Amer. Math. Soc. (to appear).

6. R. Wheeden, On the radial and nontangential maximal functions for the disc, Proc. Amer. Math. Soc. 42 (1974), 418-422.

DEP ARTMENT OF MATHEMATICS, UNIVERSITY OF CHICAGO, CHICAGO, ILLINOIS 60637

DEPARTMENT OF MATHEMATICS, RUTGER S UNIVERSITY, NEW BRUNSWICK, NEW JERSEY 08903 